SCIENTIFIC REPORT

\title{
Eyelid basal cell carcinoma: non-Mohs excision, repair, and outcome
}

\author{
S Hamada, T Kersey, V T Thaller
}

Br J Ophthalmol 2005;89:992-994. doi: 10.1136/bjo.2004.058834

Aim: To analyse the outcome of basal cell carcinoma (BCC) excision in a subregional (non-Mohs) oculoplastic service. Methods: A single surgeon retrospective series, medical record review of 223 consecutive cases with histologically confirmed eyelid BCC (between 1987 and 2004). Tumour recurrence rate was derived from the 69 patients with a minimum 5 year follow up.

Results: Of the total 223 patients, the surviving 162 were included in this study. The follow up for the whole cohort ranged from 2 months to 120 months. All cases underwent excisional biopsy. $4 \mathrm{~mm}$ excision margins were taken in $83 \%$ of cases. The pathology revealed $84 \%$ complete primary excision. Of those reported incompletely excised 53\% contained no tumour at re-excision. $70 \%$ of lid defects were treated by primary direct closure. Following confirmed histological clearance the remainder underwent delayed direct closure (2\%), full thickness skin or tarsal grafts (13\%), local skin and muscle flaps (11\%), and spontaneous granulation (laissez faire) (4\%). No major complications were noted. There were no recurrences for non-infiltrative BCCs. The overall 5 year and over recurrence rate including previously recurrent $\mathrm{BCC}$ s was $4.35 \%$, only one of which was in the primary $\mathrm{BCC}$ group (1.6\%). All recurrences were in infiltrative BCCs.

Conclusions: Non-infiltrative BCC excision with $4 \mathrm{~mm}$ margins gave a zero recurrence rate. Long term follow up of such patients may be unnecessary. Infiltrative BCCs should be followed up indefinitely. Previous recurrence and infiltrative histology have predictive value for recurrence. We achieved one of the lowest recurrence rates reported in non-Mohs surgical excision. Direct closure was applicable in $72 \%$ of cases.

B asal cell carcinoma (BCC) is the most common skin cancer. Almost $90 \%$ occur on the head and neck with $10 \%$ of those involving the eyelid. ${ }^{1-3}$ BCCs are slow growing, non-metastasising, malignant tumours accounting for less than $0.1 \%$ of patient deaths, but may cause major complications. ${ }^{3}$ The aim of treatment is total tumour eradication with the smallest recurrence risk, employing the most cost effective method that is acceptable to the patient. ${ }^{4}$ Mohs micrographic surgery (MMS), a method of tumour excision with complete frozen section margin control offers the lowest recurrence rate for BCC and is the standard against which other treatments are compared. ${ }^{5}$ However, it is costly, time consuming, and not generally available in the United Kingdom.

\section{METHODS}

We reviewed case notes and histology from a consecutive, single surgeon (VTT) series of patients operated on between

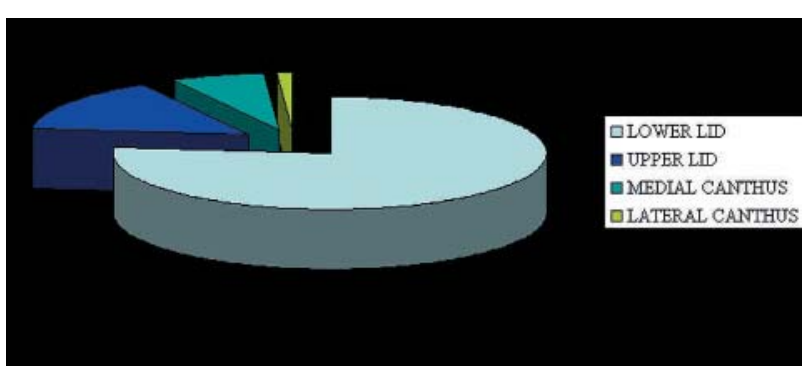

Figure 1 BCC lid location.

1987 and 2004. All BCC types and tertiary referrals with recurrent tumours were included.

Tumour margins were marked on stretch using the following guides:

- Transition in surface contour (BCCs often elevated)

- Altered vascularisation (BCCs have fewer capillaries and more telangectasia)

- Altered skin colour (under stretch, BCCs paler than surrounding skin)

- Alteration in surface texture by light reflection

- The deep extent of the tumour is judged by the lesion's mobility over the underlying tissues.

"Safety" margins of $4 \mathrm{~mm}$ were marked with the skin still under tension. The tumours were excised and the specimen edges dyed for orientation. Where possible well demarcated tumours were closed directly without undermining. Poorly demarcated tumours or large defects requiring more complex repair were simply padded firmly with chloramphenicol ointment and a non-stick dressing (Tegapore) while awaiting paraffin section histological confirmation of tumour clearance (2-4 days). If the tumour was found to extend to any margin of the specimen, a further excision was made from the involved margin and further histology awaited. Reconstruction was by direct closure (even under severe tension) wherever possible. Otherwise standard reconstruction techniques were employed. Sutures were removed at 1-2 weeks. Follow up was at 3-6 months, and thereafter annually for a minimum of 5 years, longer for recurrent and infiltrating tumours. Only patients with a minimum 5 year follow up period were used for tumour recurrence analysis.

\section{RESULTS}

In all, 223 patients with BCC were treated between 1987 and 2004, representing 54\% of all eyelid BCCs treated in our trust. The surviving 162 patients were included. All deaths were

Abbreviations: $B C C$, basal cell carcinoma; MMS, Mohs micrographic surgery 


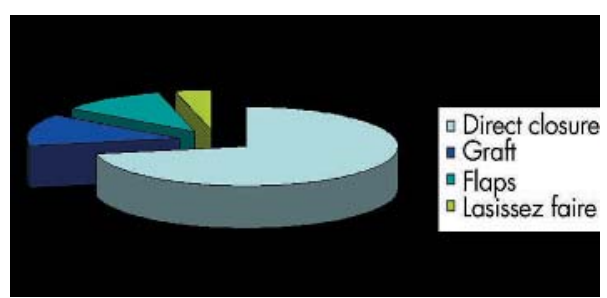

Figure 2 Methods of lid reconstruction employed.

unrelated to BCC. The mean follow up was 48.6 months (range 2-120 months), the female to male ratio 1.1:1 (85:77), the mean age 71.2 (33-97) years. A total of 124 tumours were on the lower lid $(76.5 \%), 25$ on the upper lid $(15.4 \%), 11$ at the medial canthus $(6.8 \%)$, and two at the lateral canthus (1.2\%) (fig 1). There was equal left:right distribution.

All tumours underwent primary excisional biopsy with $4 \mathrm{~mm}$ excision margins in $83 \%, 3 \mathrm{~mm}$ in $4 \%$, and $2 \mathrm{~mm}$ in $13 \%$ of cases (the smaller margins in small, nodular tumours). Pathology confirmed $84 \%$ complete removal at primary excision. Of those reported incompletely excised 53\% contained no tumour at re-excision.

Seventy per cent of lid defects were treated by primary direct closure; none was subsequently found to be incompletely excised. The remainder, following confirmed histological clearance, underwent direct closure $(2 \%)$, full thickness skin or tarsal grafts with skin flap (13\%), local skin and muscle flaps $(11 \%)$, or spontaneous granulation (laissez faire) (4\%) (fig 2).

Histological analysis of the specimens showed $23.8 \%$ with an infiltrative growth pattern (fig 3),

There were seven pigmented and mixed nodular/infiltrative cases.

Of the few minor complications reported, granulomas $(4.1 \%)$ and trichiasis $(2.7 \%)$ were the most frequent.

Our 5 year recurrence rate for non-infiltrating BCCs was zero. There were three recurrences $(4.35 \%)$, all in infiltrative tumours (one primary and two recurrent infiltrative BCCs).

\section{DISCUSSION}

The cohort includes both primary and recurrent BCCs, including tertiary referrals from other ophthalmic and nonophthalmic colleagues.

Surgical excision is the gold standard for BCC treatment among oculoplastic surgeons, giving histological diagnosis and tumour clearance confirmation with minimal collateral damage. The safety margin of clinically uninvolved tissue, taken to minimise recurrence, significantly increases the surgical tissue defect. The actual tumour edge may be difficult to determine clinically. A 1997 study showed that surgeons' estimations can vary $5.5 \mathrm{~mm}$ from the actual margin of the lesion for primary BCCs. ${ }^{2}$ Tissue conservation remains an important issue in eyelid tumour surgery. To our knowledge, only one study showed no recurrences with $2 \mathrm{~mm}$ margins, but this included only nodular BCCs and a significant proportion of patients required second excisions before repair. ${ }^{6}$ We generally favour a $4 \mathrm{~mm}$ safety margin and it is our practice to carry out primary excisional (rather than incisional) biopsy to minimise inconvenience and costs. In our series $17 \%$ had less than $4 \mathrm{~mm}$ margins as they were small, well defined tumours, typically nodular BCCs.

Our proved good results and experience in direct closure for larger lid defects ${ }^{7}$ give us a strong argument against the spurious claim that $4 \mathrm{~mm}$ margins prevent direct eyelid closure (fig 4, shows 2 months postoperative results of direct closure). Consideration of ultimate defect size must never compromise the goal of complete, primary, tumour excision.

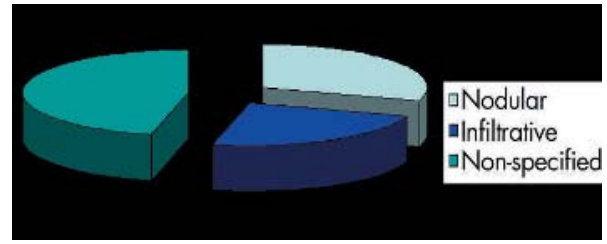

Figure 3 Histological classification.

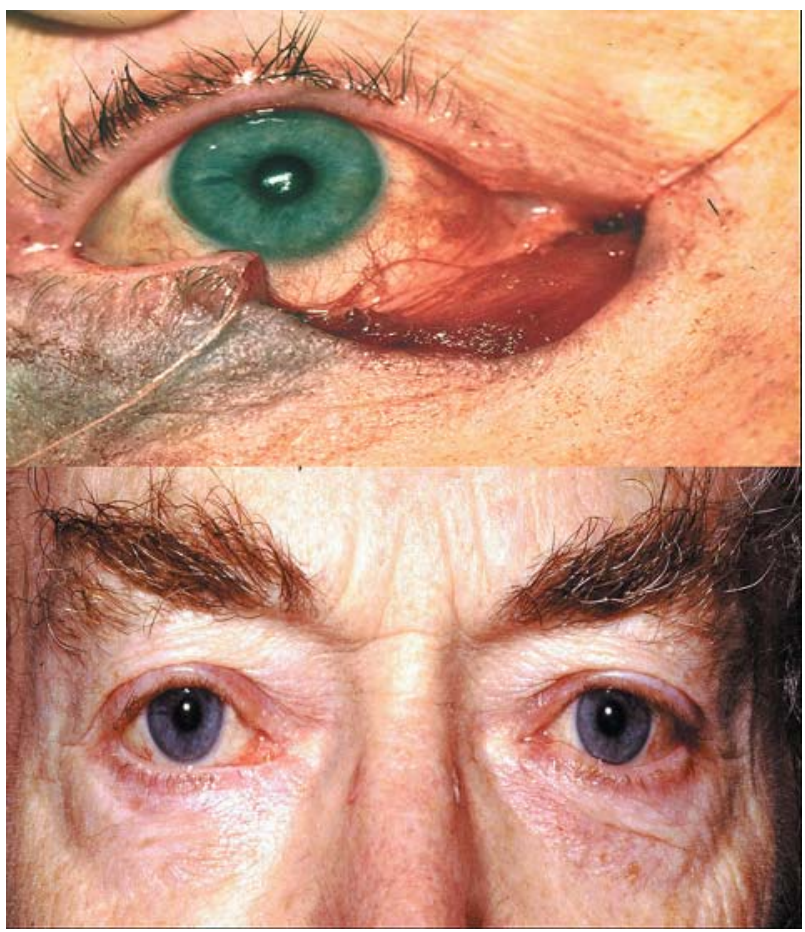

Figure 4 Right lower lid surgical defect following BCC excision and 2 month post horizontal direct closure lid reconstruction.

It has been shown that full thickness eyelid defects of up to $26 \mathrm{~mm}$ could be closed in selected patients by direct closure under tension and end up with excellent functional and cosmetic results. In this series we have been able to close lid defects directly in $72 \%$ of cases without cantholysis or undermining. This figure compares very favourably with the major BCC studies. In the Swedish study, flaps and grafts were used in 62 out of 64 patients. ${ }^{8}$

Our pathology reports revealed $84 \%$ complete primary excision. Of those reported incompletely excised, 53\% contained no tumour at re-excision, which is in agreement with previous studies.

We know that only a minority of patients with histologicaly documented positive margins will experience a recurrence. ${ }^{9}$ DeSilva and Dellon ${ }^{10}$ followed 38 patients with positive margins and only $37 \%$ recurred. Gooding ${ }^{11}$ reported a $35 \%$ recurrence rate and Shanoff ${ }^{12}$ reported $67 \%$. Sarma et al suggest that tumour cells at the operative site may be devitalised by surgery, thus accounting for the lower than expected recurrence rate. ${ }^{13}$ An alternative, more likely explanation is that the margins are so narrow that the specimen distortion through fixing and tissue processing and sampling makes it difficult for the pathologist to confidently confirm clearance. Hauben ${ }^{14}$ found a recurrence rate of $25.6 \%$ with positive margins and $22.8 \%$ with negative margins. The question remains whether to do further surgical excision if the completeness of the initial excision is in doubt. 
Conventional methods used to determine margin adequacy do not allow for the discontinuous appearance of BCC in histological sections. Recurrence rates can be distressingly high even in lesions with "negative" surgical margins. Lang ${ }^{15}$ studied 10 recurrent tumours, six of which had negative margins with the initial excision.

Our 5 year minimum follow up group showed a zero recurrence rate for non-infiltrative BCCs. Therefore, for this group long term follow up might not be necessary. Our overall recurrence rate was $4.35 \%$ with all three recurrences being in infiltrative cases. One occurred in the primary BCC group while the other two were already recurrent cases. One occurred after 8 years, highlighting the importance of long follow up for these cases. Interestingly, all the recurrent cases reported postoperative complications following the primary excision: entropion, trichiasis, and dry eye. Our follow up time ranged from 2-120 months. Prospective studies have shown that $36 \%$ of patients who develop BCC will develop a second primary BCC within the next 5 years. ${ }^{3}$ Steinkogler et al emphasised the importance of long term follow up for tumours that show large horizontal and depth extension, exceptional height, and especially secondary tumours. ${ }^{16}$

The majority of our patients ended up with excellent functional and cosmetic results. There were only minimal surgical complications such as granuloma formation $4.1 \%$ and trichiasis $2.7 \%$ during the follow up period. These finding confirmed the positive results of the direct lid closure study, the reconstruction technique of choice, used in $72 \%$ of this series. Complications occurred more often in cases that needed large lid excision and hence complicated surgical repair. Similar findings were noticed in the Swedish Mohs study. ${ }^{17}$

Finally, the Australian Mohs micrographic surgery database, which is the largest prospective series of periocular BCC managed by MMS, concluded that MMS is the treatment of choice for periocular BCC. ${ }^{18}$ We consider this impractical for most lid BCCs on cost/benefit grounds as well as the difficulty of obtaining a true Mohs layer in inhomogeneous lid tissue. Our conventional (non-Mohs) technique provides reliable and cost effective surgical treatment for BCCs.

\section{CONCLUSION}

Non-infiltrative BCC excision with $4 \mathrm{~mm}$ margins gave a zero recurrence rate; long term follow up may therefore be unnecessary. Infiltrative or previously recurrent BCCs should be followed up indefinitely (time to recurrence 6-98 months).
Repair by direct closure was achievable in $72 \%$ of cases giving excellent functional and cosmetic results and should be attempted whenever possible.

We achieved one of the lowest recurrence rates reported in non-Mohs surgical excision series.

\section{Authors' affiliations}

S Hamada, T Kersey, V T Thaller, Royal Eye Infirmary, Plymouth, UK

Correspondence to: V T Thaller, FRCOphth, The Royal Eye Infirmary, Apsley Road, Plymouth PL4 6PL, UK; vladimir.thaller@phnt.swest.nhs.uk

Accepted for publication 4 January 2005

\section{REFERENCES}

1 Salomon J, Bieniek A, Baran E, et al. Basal cell carcinoma on the eyelids: own experience. Dermatol Surg 2004;30:257-63.

2 Goldberg DP. Assessment and surgical treatment of basal cell skin cancer. Clin Plast Surg 1997;24:673-86.

3 Duong HQ, Copeland R. Basal cell carcinoma, eyelid. Emedicine 2001.

4 Anthony ML. Surgical treatment of nonmelanoma skin cancer. AORN J 2000;71:552-8, 560.

5 Malhotra R, Huilgol SC, Huynh NT, et al. The Australian Mohs database, part I: periocular basal cell carcinoma experience over 7 years. Ophthalmology 2004;111:624-30

6 Hsuan JD, Harrad RA, Potts MJ, et al. Small margin excision of periocular basal cell carcinoma: 5 year results. Br J Ophthalmol 2004;88:358-60.

7 Thaller VT, Then KY, Luhishi E. Spontaneous eyelid expansion after full thickness eyelid resection and direct closure. $\mathrm{Br} J$ Ophthalmol 2001;85:1450-4

8 Lindgren G, Lindblom B, Larko $O$. Mohs' micrographic surgery for basal cell carcinomas on the eyelids and medial canthal area. II. Reconstruction and follow-up. Acta Ophthalmol Scand 2000;78:430-6.

9 Seidman JD, Berman JJ, Moore GW. Basal cell carcinoma: importance of histologic discontinuities in the evaluation of resection margins. Mod Pathol 1991;4:325-30.

10 De Silva SP, Dellon AL. Recurrence rate of positive margin basal cell carcinoma: results of a five-year prospective study. J Surg Oncol 1985;28:72-4.

11 Gooding CA, White G, Yatsuhashi M. Significance of marginal extension in excised basal-cell carcinoma. N Engl J Med 1965;273:923-4.

12 Shanoff LB, Spira M, Hardy SB. Basal cell carcinoma: a statistical approach to rational management. Plast Reconstr Surg 1967;39:619-24.

13 Sarma DP, Griffing CC, Weilbaecher TG. Observations on the inadequately excised basal cell carcinomas. J Surg Oncol 1984;25:79-80.

14 Hauben DJ, Zirkin H, Mahler D, et al. The biologic behavior of basal cell carcinoma: analysis of recurrence in excised basal cell carcinoma: Part II. Plast Reconstr Surg 1982;69:110-16.

15 Lang PG Jr. Maize JC. Histologic evolution of recurrent basal cell carcinoma and treatment implications. J Am Acad Dermatol 1986;14:186-96.

16 Steinkogler FJ, Scholda CD. The necessity of long-term follow up after surgery for basal cell carcinomas of the eyelid. Ophthalmic Surg 1993:24:755-8.

17 Lindgren G, Lindblom B, Larko O. Mohs' micrographic surgery for basal cell carcinomas on the eyelids and medial canthal area. II. Reconstruction and follow-up. Acta Ophthalmol Scand 2000;78:430-6.

18 Malhotra R, Huilgol SC, Huynh NT, et al. The Australian Mohs database. Part II: Periocular basal cell carcinoma outcome at 5-year follow-up. Ophthalmology 2004;111:631-6. 